\title{
LA IMPORTANCIA DEL ENFOQUE DE GÉNERO EN LA INVESTIGACIÓN SOCIO-HISTÓRICA
}

\section{THE IMPORTANCE OF GENDER IN SOCIO-HISTORICAL RESEARCH}

\author{
Nancy Piedra Guillén*
}

RESUMEN

El siguiente artículo se inscribe en el debate de los estudios feministas que se proponen hacer una historia inclusiva en donde las mujeres, por su condición y posición, no queden relegadas al margen de los hechos sociales que tienen lugar en el tiempo y el espacio; que la mirada androcéntrica no gane la batalla y que esos hechos, algo invisibles a simple vista, puedan ser observados, analizados y revelados. En fin, que la genealogía de los procesos históricos, tal cual lo señala Foucault, permita ir más allá para descubrir lo que la historia oficial no ha descubierto, o bien, ha velado y omitido por su poca o mucha trascendencia. Se pretende plasmar en este artículo, una entrada que retoma varios aspectos teóricometodológicos que en sí mismos no son novedosos, de modo que el aporte consiste en cómo se relacionan estos constructos, cómo se articulan entre sí para avanzar y contribuir con el desarrollo de las reflexiones, en particular, la participación de las mujeres en hechos socio-históricos.

PALABRAS CLAVE: HISTORIA * MUJERES * GÉNERO * IDENTIDAD * VIDA COTIDIANA * POSTCOLONIALISMO

\section{ABSTRACT}

The following article is based on the debate of feminist studies which purpose is to make an inclusive history where women and minorities for their condition and position are not at the margin of social facts that pass by in time and space, so that the androcentric view doesn't win the battle, and that those acts somewhat hidden, or not visible at plain view, could be discovered, observed, analyzed and revealed. The genealogy of historic processes, as outlined by Foucault, would allow to go beyond what official history has not shown or has not told, or let by the wayside, due to its degree of significance.

We want to capture in this article, an aspect that takes several theoretical methodological what themselves are not new, so the contribution is how to relate these constructs, how they articulate with each other. In particular, in socio-historical facts, the participation of women.

KEYWORDS: HISTORY * WOMEN * GENDER * IDENTITY * EVERYDAY LIFE * POSTCOLONIALISM

Escuela de Sociología de la Universidad de Costa Rica (UCR).

npiedraguillen@yahoo.com 


\section{INTRODUCCIÓN}

¿Cómo analizar lo intangible, observar lo no visto, estudiar lo no estudiado? Esta es en parte la labor que debe enfrentar toda persona que trabaja desde la perspectiva sociohistórica, más aún si desea estudiar la presencia de las mujeres y de las relaciones de género. Se debe destacar el desarrollo de estudios que consideren a las mujeres como sujetos histórico sociales, para ello es necesario analizar ¿en qué consisten las relaciones entre los sexos, los géneros?, ¿cuáles son los papeles que hombres y mujeres tienen en una sociedad determinada?, ¿cómo se define la feminidad desde el estatus - posición- de las mujeres? Un enfoque que considere estas inquietudes:

... modifica la visión de lo que se entiende tradicionalmente como historia ( $y$ el ejercicio del poder) y modifica también las premisas de las categorías de análisis histórico, al privilegiar como objeto de análisis la relación entre los géneros. Las relaciones entre los sexos y el género se convierte entonces en una categoría social, en una construcción social dada en un tiempo y espacio determinados, $y$ no en una relación condicionada y predeterminada por la biología (Ramos, 1992: 13).

La historia de las mujeres se presenta, en alguna medida, como justificación de su propia legitimación, en tanto rama de la historia y evoluciona hasta alcanzar los planteamientos avanzados por la historiografía consolidada $y$ reconocida en la actualidad.

La historia de la mujer es una corriente que desarrolla sus líneas de investigación con la elaboración de nuevos marcos conceptuales o reconceptualizaciones de estos, basada en una metodología que busca nuevas fuentes $y$ documentación $y$ el replanteamiento de las tesis históricas tradicionales a partir de este nuevo bagaje y metodología (Nash, 1984: 11).

Con la historia de las mujeres se pretende observar y analizar las aportaciones que los géneros han hecho al proceso histórico, concebido como el conjunto de experiencias acumuladas de los varones y de las mujeres; hasta hace pocos años solo se observaban con especial interés las acciones realizadas por los hombres, en tanto se entendía que la historia respondía a los vencedores y no a los vencidos, sin prestarle atención a las acciones concretas que desarrollaban las mujeres y que no necesariamente correspondían a las ejecutadas por los hombres. Así, en este artículo se procura señalar algunos aspectos conceptuales de este enfoque, el cual ha generado debates en otros países de Europa y Estados Unidos, pero menos presente en Costa Rica, en donde recientemente se retoma a pesar de la producción historiográfica que existe con respecto a las mujeres.

Esta discusión teórica-metodológica toma un auge particular a raíz del desarrollo de la teoría postcolonial y con ella, del feminismo poscolonial, postura que posibilita retomar, reconstruir y apropiarse desde las periferias, el pensamiento del feminismo occidental para avanzar en nuestro propio desarrollo analítico. Por ello, se considera que el siguiente artículo aporta aspectos teórico-metodológicos que son sustanciales para el desarrollo de la investigación historiográfica de las mujeres en Costa Rica.

\section{GÉNERO Y CAMBIO}

Realizar un estudio con enfoque de género es analizar las prácticas sociales y culturales de hombres y mujeres en un periodo socio histórico determinado. El desarrollo de nuevas prácticas, tanto en varones como en mujeres, está relacionado con los procesos de construcción identitaria.

La identidad de cada "nosotros" depende de una estructura de poder; las colectividades se constituyen a sí mismas no solo excluyendo, sino también oprimiendo a otros por encima $y$ contra quienes se definen. Al respecto, Benhabib señala: "En este sentido, la identidad de cada "nosotros" contiene el resultado de luchas colectivas por el poder entre grupos, culturas, géneros y clases sociales" (1996: 31).

Después de décadas, al menos tres, de fructífera producción analítica, tanto teórica como empírica, el estudio de las relaciones de 
género y el análisis de las identidades sexuales $y$ de género, ha re-abierto debates teóricos en torno a cómo se construye la identidad de género - masculina y femenina- y si esta visión dicotómica no es más bien limitada para comprender su complejidad. Se parte, como punto de confluencia, de que las identidades, tanto personales como colectivas, son construcciones sociales y no están determinadas biológicamente, más dicha afirmación no da cuenta de la realidad en su totalidad.

El género, como categoría de análisis, enfatiza la necesidad de distinguir algunos términos para evitar confusiones en nuestra interpretación y captar de mejor forma la realidad. Así, es necesario diferenciar entre:

... el sexo construido en términos biológicos, la sexualidad entendida como algo que abarca prácticas sexuales y comportamientos eróticos, la identidad sexual referida a designaciones como heterosexuales, homosexuales/gay/lesbiana/ queer, bisexual o asexual; la identidad de género como un sentido psicológico de uno mismo como hombre o como mujer; el papel de género como un conjunto de expectativas prescriptivas y específicas de la cultura sobre qué es lo apropiado socialmente en hombres $y$ en mujeres; $y$ la identidad del papel de género, como un concepto ideado para captar en qué medida una persona aprueba y comparte sentimientos $y$ conductas que se consideran que son apropiados a su género constituido culturalmente (Hawkesworth, 1999).

Desde este contexto, no es casual que en la década del 90 del siglo xx, a la luz del desarrollo de dos corrientes de análisis - el movimiento por la paridad y el feminismo postcolonial-, el debate tomara centralidad.

El movimiento por la paridad buscó redefinir el universalismo francés con el fin de aumentar el número de mujeres en puestos de representación política. Durante años, se demandó la escasa presencia de las mujeres en puestos de poder político, pese a que por más de medio siglo las mismas habían adquirido el derecho al voto. Al respecto, la filósofa Françoise Collin señala que: "fue el universalismo el que contribuyó a mantener la sexualización del poder, y que la paridad, por el contrario, pretende desexualizar el poder al extenderlo a ambos sexos. La paridad podría ser entonces, el verdadero universalismo" (2006: 21).

Así, dos posturas teóricas explicativas sobre la identidad siguen teniendo gran influencia en la teoría feminista contemporánea, universalismo vs. diferencia ${ }^{1}, y$ generan un debate para comprender las diferencias, las desigualdades e inequidades que entraña la relación de las mujeres con los "otros".

El feminismo cultural asume que es posible partir de una definición previa del "concepto mujer". Las mujeres pueden ser definidas partiendo de sus actividades y atributos en la cultura actual, los cuales se han mantenido en el tiempo. Por ejemplo, el patriarcado es una relación de dominación de los hombres sobre las mujeres que se mantiene en la historia, a pesar de las transformaciones que se dan en el tiempo. Así, el patriarcado se crea y se instituye con el derecho romano, pero cambia en el tiempo, en algunas sociedades se agudiza la relación de dominación y en otras el poder masculino se relativiza. Por su parte, la posición del feminismo posestructuralista parte de que no hay un concepto de "mujer", definido o definible 2 .

1 "Desde sus comienzos en el siglo XVIII y más particularmente en el período de su articulación a mediados del siglo XIX y principios del XX, el feminismo y los movimientos de mujeres siempre se han debatido con los dilemas de la igualdad y la diferencia: igualdad con los varones versus diferenciarse respecto a ellos; preservar una esfera separada de mujeres versus convertirse en miembros plenos de la sociedad existente, abandonando los espacios tradicionales de las mujeres" (Benhabid, 1996: 25). A su vez el feminismo de la diferencia plantea, en oposición al feminismo de la igualdad, que las mujeres tenemos múltiples identidades, las cuales no se pueden reducir solo al género - esta es vista como una categoría más- (Vargas, 1998), mientras que el feminismo de la igualdad parte del principio central de que todas las mujeres somos iguales, en tanto mujeres todas pertenecemos a un mismo estatus de inferioridad con respeto a los hombres.

2 Si se desea profundizar en la temática, el artículo de Piedra (2003) desarrolló un análisis sobre las distintas corrientes, con el objetivo de profundizar en el estudio de las feministas de la diferencia. 
Las teóricas posmodernas consideran que no se debe apelar a aspiraciones universalistas sobre la situación social de las mujeres (Piedra, 2003). Es necesario mirar, estudiar e identificar las diferencias porque:

... la afirmación del universal "mujer" puede ser el vehículo de dominación de una parte de las mujeres, que ocupan situaciones de privilegio relativo, sobre el resto, o un modo de conseguir alianzas en la defensa de intereses particulares apelando a unos supuestos intereses universales (Izquierdo, 1998: 37).

Por otro lado, una de las críticas que se hace al movimiento por la paridad es el énfasis que pone en las diferencias de género, dicotomizándolas y sexualizándolas. La filósofa Sylviane Agacinsky, se apartó del movimiento por la paridad cuando en lugar de sexuar al individuo abstracto se pasó a asexuar al político, como lo hacían las fundadoras del movimiento, por lo cual propuso sexuar el cuerpo político por la complementariedad de la pareja heterosexual y volvió a la idea de la diferencia sexual como incompatible $y$ antagonista con el individualismo abstracto. Hay quienes han desarrollado explicaciones alternativas a dichas posturas teóricas con el fin de dar respuesta a las limitaciones de las explicaciones pos-estructuralistas y culturalistas, como es el caso de Linda Alcoff, quien propone el concepto de posicionalidad, planteando que la identidad de una mujer es producto de su propia interpretación y reconstrucción de su historia, a través del contexto discursivo cultural al que tiene acceso.

El concepto de mujer según la 'posicionalidad', muestra cómo las mujeres usan su perspectiva posicional como un sitio desde el cual se interpretan y construyen los valores, más que el lugar de un conjunto ya determinado de valores, visión de mundo y estructuras que constriñe a la persona (Alcoff, 1989: 14).

En América Latina, este debate no ha sido ajeno al interés de las investigadoras feministas, razón por la cual en los estudios acerca de la construcción de identidad(es), se pueden encontrar dichas diferencias teóricas. Dentro de la corriente de la posicionalidad se ubica a estudiosas como María Luisa Tarrés (1997), Florinda Riquer Fernández (1997), Tine Davids, Teresa de Lauretis (1987). Por ejemplo, Lauretis plantea que la subjetividad no está sobredeterminada por la biología ni por la intencionalidad libre y racional, sino por la experiencia entendida de cada sujeto: experiencia entendida como un complejo de hábitos resultantes de la interacción con el mundo externo.

En otra corriente de análisis se encuentran los trabajos de Marcela Lagarde, quien desde una concepción culturalista (con énfasis en aspectos antropológicos e históricos estructurantes) plantea el problema de las mujeres, en tanto sujetas sociales que han vivido en un "cautiverio histórico" (Lagarde, 1997: 68) ${ }^{3}$, producto de la relación de jerarquía superior que tiene el género masculino en relación con el femenino (Lagarde, 1993: 55) ${ }^{4}$.

Se encuentra en el medio de estas dos posturas, la propuesta teórica performativa de las identidades de género de Butler, quien se propuso en su libro El género en disputa, tal y como ella misma afirma, cuestionar un supuesto heterosexual dominante en la teoría literaria feminista:

3 Para Marcela Lagarde, el cautiverio obedece a la condición jerárquicamente inferior de las mujeres con respecto a los hombres. "Ser hombre implica vivir desde una condición de género privilegiada, jerárquicamente superior y valorada positivamente. Y ser mujer implica vivir a contracorriente, desde la condición inferiorizada a partir de la cual los hechos de las mujeres son desvalorizados o invisibilizados, $y$ las colocan de antemano en una condición jerárquica menor, subordinada, y sometida a dominación. En síntesis la condición política de las mujeres en el mundo patriarcal es el cautiverio y la de los hombres es de dominio".

4 Lagarde señala que tenemos una organización muy esquemática de la identidad, por ello es que se puede ser muy pocas cosas a la vez, además de que estamos determinados históricamente para ser de ciertas maneras y no de otras. De esta manera, en un sistema de especializaciones para vivir, unas somos especializadas mujeres $y$ otros son especializados hombres. Las identidades femeninas y masculinas no son fenómenos naturales sino hechos construidos social e históricamente. 
Mi postura era y sigue siendo que cualquier teoría feminista que restrinja el significado del género en las presuposiciones de su propia práctica establece normas de género excluyentes en el seno del feminismo, a menudo con consecuencias homofóbicas (Butler, 2001).

Para desarrollar su constructo explicativo, se retoma a varios(as) intelectuales franceses como Levi Strauss, Foucault, Lacan, Kristeva y Wittig (Butler, 2001). Se considera que cada cual le permite abordar al tema de fondo, la jerarquía del género con algunos aspectos específicos. Esta jerarquía del género, que a su vez responde a una heterosexualidad más o menos obligada, fundada en la visión dicotómica del concepto de género. Pero "¿queda algo que se llame cuerpo mismo que escape a toda esquematización?" (Butler, 2001: 90). Desde su perspectiva existen dos respuestas: la psique como dadora de unidad al cuerpo y la psique como aquello que constituye el modo en que se da o se determina el cuerpo. Así, el cuerpo "es aquello sin lo cual" no podría darse ninguna operación psíquica, pero es a la vez aquello que sobre $y$ a través de lo cual también opera la psique, es "la demanda constitutiva que moviliza la acción psíquica desde el comienzo" (Butler, 2001: 90). Al respecto, señala Torricella (2009), que en la postura de Butler existe una relación imprecisa $y$ tensa entre la performatividad $y$ la materialidad. Pero desde nuestro punto de vista, tiene la virtud de preocuparse por comprender la construcción de los significantes, no como productos dados, estructurantes, sino en constante transformación en el tiempo, en el espacio, históricamente construidos, pero no históricamente determinados.

Se puede concluir que el tema de la construcción de las identidades sexuales se mantiene abierto, retroalimentado con planteamientos diversos que permiten comprender su complejidad y la incidencia que las relaciones de desigualdad entre hombres $y$ mujeres tienen en la vida cotidiana, en las prácticas de las personas, así como en el terreno de lo político. No cabe duda de que, a su vez, el desarrollo del feminismo postcolonial ha venido a enriquecer estos debates y análisis, elemento que se retomará más adelante.

\section{GÉNERO, HISTORIA Y PODER}

Considerando el debate antes expuesto, se retoma el tema del género y la historiografía, necesario para considerar aspectos de carácter teórico-metodológico que se proponen en este artículo. Señala Scott que la diferencia sexual está basada en relaciones jerárquicas que atañen a una distribución desigual del poder: "el género es un elemento constitutivo de las relaciones sociales basadas en las diferencias que distinguen los sexos y el género es una forma primaria de relaciones significantes de poder" (Scott, 1990: 44). Es decir, el género es el campo primario dentro del cual se articula el poder, aunque no es el único. En este mismo sentido señala Bourdieu que:

La "di-visión del mundo" basada en referencias a las diferencias biológicas y sobre todo a las que se refieren a la división del trabajo de procreación y reproducción actúa como "la mejor fundada de las ilusiones colectivas", en la medida en que los conceptos de género estructuran la percepción y la organización, concreta y simbólica, de toda la vida social (2000: 48).

Existe así una distribución del poder que alude a los recursos materiales y simbólicos, que a su vez integra las relaciones de género en sus percepciones y en el acceso a dichos recursos.

Desde esta perspectiva, la práctica y la experiencia que se deriva de la vida cotidiana de mujeres $y$ varones ubicados en contextos socio históricos específicos es central, ya que permitiría acercarnos a aquellas acciones recurrentes que se remiten, a su vez, a los hábitos resultantes de la interacción entre el mundo interno y externo de los(as) sujetos(as) ${ }^{5}$.

5 Desde la perspectiva de Bourdieu, el "habitus" representa aquellas prácticas particulares que responden a condiciones de existencia. Son disposiciones "verdaderas" para un determinado contexto que a su vez son transferibles; en sí, principios generadores y ordenadores de prácticas. 
Salles señala que no se debe dejar de lado que el orden social y las desigualdades de género que se derivan de este mismo, no se agotan en las relaciones, en las formas de convivencia $y$ en las experiencias que lo fundan: un orden social también se sustenta en las instituciones, que a pesar de originarse en la actividad humana rutinizada (según Giddens) o en la acción habitualizada (según Bourdieu, 1999 $y$ Wacquant, 2002), adquieren fuerza propia en tanto suelen determinar los espacios, las interacciones $y$ las relaciones que en ellas se pueden desarrollar, obedeciendo así al "orden" o exigencias que demanda cada sociedad, mas ello no implica que las acciones de los sujetos puedan modificarlas abruptamente transformando paulatinamente las estructuras.

Se deriva de esta perspectiva de género que aunque toda relación entre los géneros, así como, las restantes relaciones sociales, están mediadas por relaciones de poder, ello no implica que estas no puedan ser modificadas: de hecho, las relaciones de poder - género y sociales- han cambiado en el curso de la historia y ello se debe a los procesos de transformación que se producen en cada sociedad, mediados en parte por las acciones $y$ el ejercicio de nuevas prácticas que las personas realizan en cada contexto social particular.

Se piensa que a diferencia de lo antes señalado, existen otras conceptualizaciones ahistóricas de las relaciones de poder entre los géneros, que tienden a generalizar experiencias de unas mujeres a las restantes. Dichas perspectivas impiden analizar las particularidades, así como analizar los procesos de cambio, las rupturas y las diferencias. Al respecto, Scott (1990) recuerda la importancia de rechazar los orígenes sencillos para dar pie al análisis de procesos interrelacionados que no puedan deshacer sus nudos.

Metodológicamente, Scott plantea considerar en el análisis cuatro dimensiones que deben ser incorporadas para abordar los estudios

El habitus es sistemático y transponible, es decir, puede transponerse de un ámbito de práctica a otro. Es un esquema que existe en estado práctico, por lo tanto, se le interioriza de modo implícito, pre reflexivo y pre-teórico (Bourdieu, 2000). de género, en tanto relaciones sociales y de poder. Estos aspectos son reconocidos aún en la historiografía feminista pues se considera que han marcado el desarrollo de la historia de las mujeres y del movimiento feminista y que siguen teniendo validez (Borderías, 2006). Las dimensiones de análisis que plantea Scott en la década de los 80 del siglo pasado, tienen la particularidad de ser teórico-metodológicas, ello permite aprender $y$ comprender las diversas expresiones de la realidad socio-histórica ${ }^{6}$.

La pregunta central que surge, continuando con inquietudes expresadas en el apartado anterior, es cómo se construyen las identidades genéricas y cómo se relaciona dicho aspecto con las actividades, las organizaciones sociales y las representaciones culturales históricamente específicas. Sin embargo, a finales de los años 90 y a inicios del siglo Xx, Scott confesaba que cada vez utilizaba menos el concepto de género en su trabajo (Aresti, 2006), ya que "género" había perdido su carga crítica y a veces se le utilizaba como sinónimo de mujeres o sexo. Según lo ha expresado ella misma, estamos en la necesidad de crear nuevos términos que permitan comprender y explicar la realidad como historiadoras(es) o científicas(os) sociales. Pero, años más tarde, la misma Scott (2006), analizó retrospectivamente su pregunta inicial, ¿es el género una categoría útil para el análisis historiográfico? En ese sentido, apunta que en 1986, cuando ella planteó el tema en American Historical Review, la mayoría de los y las colegas presentes no estaban preparados para ello, para el género, ni para la teoría postestructuralista, pero a su vez, comprendió que su análisis representaba un esfuerzo colectivo del que ella formaba parte y ello le ha permitido permanecer en el

6 Las cuatros dimensiones son: 1) La dimensión simbólica: esta evoca representaciones múltiples, es decir, mitos socialmente construidos. 2) La dimensión normativa: representa las interpretaciones de los significados de los símbolos. Se expresan en doctrinas religiosas, educativas, científicas, legales y políticas que a su vez, afirman el significado de varón y mujer, masculino y femenino. 3) La dimensión sistémica: hace referencia a las instituciones $y$ organizaciones sociales como es el sistema de parentesco, educativo, económico y político. 4) La cuarta dimensión hace referencia a la identidad subjetiva. 
tiempo. Después de conocer múltiples trabajos, considera $-y$ he aquí lo más importanteque: "el género sigue siendo una categoría útil para el análisis histórico" (Scott, 2006: 109), siendo una pregunta abierta para comprender los significados y los contextos en que se hacen.

A continuación la explicación que en esta ocasión plantea:

Yo sostendría que el género es el estudio de la relación (en torno a la sexualidad) entre lo normativo y lo psíquico, el intento de colectivizar la fantasía y al mismo tiempo usarla para algún fin político o social, sin importar que ese fin sea la construcción de una nación o la estructura familiar. Mientras tanto, es el sexo género el que produce significados del género... si es así, entonces no sólo no hay distinción entre el sexo y el género, sino que el género es la clave del sexo (Scott, 2009: 109).

Por esta razón, considera que el género sigue siendo útil, puesto que es la forma de historizar las formas en que se ha concebido el sexo y la diferencia sexual. Además se resalta la importancia que da a lo psíquico, que da pie a la imagen, a la representación social, a la fantasía, en fin, a las construcciones del género.

\section{GÉNERO E HISTORIA}

Una vez señalada la relación entre el género, el poder y la historia, se puede retomar la necesidad de estudiar cuál ha sido la presencia del género femenino o de las mujeres en el acontecer histórico o el protagonismo histórico, ya que a pesar de que el sexo femenino ha representado la mitad o más de la población humana, las diversas corrientes historiográficas, tanto tradicionales como renovadoras, han excluido a las mujeres de sus estudios (Nash, 1998). Por lo tanto, se parte de que las mujeres necesitan reconstruir su historia porque:

El desconocimiento del pasado de un grupo social implica ignorar su acción histórica, bloquear el camino para la adquisición de conciencia de grupo y para la actuación en el presente en función de un futuro deseado. Dejar a un grupo sin memoria de sí mismo "conlleva una debilitación de su identidad y su anclaje en la objetivación permanente" (Vigil, s.f.: 23).

Se considera que para acceder a la recuperación histórica del género femenino, la historia de las mujeres no puede concebirse igual a la de los varones, por eso "la nueva historiografía trata de comprender el significado de los sexos, de grupos de género (gender groups) en el pasado histórico" (Zemon, 1976: 91).

El planteamiento de la historia de las mujeres, desde nuestra visión, es relevante en tanto permite vincular dos aspectos importantes en el desarrollo de la historiografía: por un lado, se cuenta con los aportes más historiográficos de la historia social —área que se ha interesado por analizar a los actores sociales en toda su dimensión-, en donde se visibilizan los sectores más marginados, de quienes no se rescataba su experiencia social e individual por no protagonizar procesos históricos de forma evidente. Esto definitivamente enriquece los procesos históricos, aceptando con ello la dinamicidad de la historia, de los perpetuos procesos, de la complejidad en que nos desarrollamos. Sin embargo, a pesar de todo ese contenido humanizador de la historia, de la historia de los hombres sencillos, de la gente de todos los días, la historia social no ha integrado en sus propuestas teórico-metodológicas la experiencia particular de las mujeres. Este error, a nuestro modo de ver, no solo lo ha cometido la historia social, todo lo contrario, los ejemplos sobran; tenemos por ejemplo, el caso de la teoría liberal; así como, la teoría marxista tradicional.

Se debe reconocer que la invisibilidad de las mujeres en los estudios históricos, sociológicos y en general, en las ciencias sociales, no corresponde a la conspiración malvada de los hombres, sino a la concepción androcéntrica de las ciencias en general. La elección del campo de estudio, su delimitación y los temas en general, obedecen a presupuestos ideológi- 
cos que determinan o influyen enormemente en los temas (Zemon, 1976: 91). La historia de las mujeres como corriente de pensamiento, es coincidente con el postcolonialismo, ya que son estudios que retoman la necesidad de conocer para de-construir las construcciones históricas que se han basado en visiones desde occidente; el objetivo principal sigue siendo "producir análisis históricos en los cuales los grupos subalternos [sean] vistos como sujetos de la historia" (Chakrabarty, 2000: 15).

Pero, desde nuestra óptica, a pesar de que la teoría como tal carece de "género", tiene en cambio un contenido androcéntrico fundado en los procesos de socialización, construcción de la subjetividad, del imaginario social y de las instituciones que en parte, norman nuestras acciones.

\section{HISTORIA, GÉNERO Y FEMINISMO POSTCOLONIAL}

Se parte de que la historia de las mujeres no se propone realizar un análisis histórico a partir de la historia total, entendida esta como el análisis sociológico del devenir histórico, en donde se consideran tanto las estructuras económicas, sociales y políticas, como los aspectos relativos a la historia privada, de las familias, las personas, la sexualidad, el trabajo doméstico, la socialización de hijos e hijas, entre otros aspectos, para establecer una visión integral del conjunto de la experiencia histórica y social de las mujeres, la cual incluya la historia de las mujeres y de género, para entender la sociedad y sus procesos de transformación desde la particularidad de la vida de estas.

De ahí que la historia de las mujeres necesite del concepto de género como categoría de análisis, perspectiva que intenta analizar las condiciones que posibilitan la subordinación de las mujeres y que para hacerlo no desdeña la intromisión en la vida privada y cotidiana de las personas, considerando a la vez la reducción dicotómica que implica el concepto y por ello, las limitaciones que puede contener, aspecto que ha motivado la reflexión constante de autoras como Scott (2006 y 2009) y Amorós (2000 y 2008).

Más recientemente $y$ en muchas ocasiones de forma paralela, el debate feminista se retroalimenta del postcolonialismo. Al relacionar ambas tendencias teóricas, se responde a la crítica que desde hace al menos dos décadas se plantea al feminismo $y$ a las distintas teorías que han generado para estudiar el desarrollo del patriarcado, la desigualdad y dominación masculina sobre las mujeres. Postcolonialismo $y$ feminismo tienen un entronque en común, tal y como lo define Linda Hutcheon: (de)construcción de la subjetividad, (de)construcción de la historia y la necesidad de la transformación social. La (de)construcción del sujeto se hace en cada cual desde distintas perspectivas, ya que el feminismo enfatiza en el sujeto patriarcal y el postcolonialismo en el sujeto imperialista ${ }^{7}$. Metodológicamente, ambos plantean la necesidad de estudiar los discursos y el cuerpo, en el que se puede encontrar respuesta a las relaciones de poder, las representaciones sociales como realidad $y / 0$ como constructo teórico. Nótese que de nuevo se da importancia al cuerpo para comprender las desigualdades de género, más sin embargo, se señala que al igual que en el discurso postmoderno, el postcolonialismo dejó de lado a las mujeres en el momento de teorizar. Al respecto, Arreaza (2002) señala que "la ausencia de una perspectiva de género en el momento de teorizar puede redundar en la perpetuación de la diferenciación jerárquica entre los sexos $y$, por extensión, en otros tipos de divisiones identitarias" (Arreaza y Tickner, 2002: 35).

De nuevo, tal y como ha pasado con otros constructos teóricos, son las feministas que retoman la reflexión postcolonial para pensar en la condición de las mujeres y desde allí, realizan un entronque entre teoría feminista $y$ el postcolonialismo. Dicha reflexión entre el postcolonialismo y el feminismo ha llevado a plantearse como centro de análisis, la dificultad que ha tenido el feminismo de incluir el cuerpo racial como objeto de estudio. En 1992, Sara Suleri planteó que uno de los peligros del feminismo occidental remite

$7 \quad$ Si desea profundizar en la temática puede consultar el texto de: Hutcheon, Linda. "Circling the Downspount of Empire". Post-Colonialism and Postmodernism 20 (4). España. Ariel, octubre 1989: 149-175. 
a la tentación de caer en la construcción de una identidad esencialista a la mujer "postcolonial" o del tercer mundo, mientras evita aplicar tal definición a la mujer occidental. Razón por la cual una de las autoras más influyentes de la teoría postcolonial, Gayatri Chakravorty, desde su condición de mujer tercermundista que vive en el primer mundo (nació en Calcuta y se traslada a vivir a Estados Unidos), combina el marxismo, el feminismo y la teoría de la deconstrucción (Carrera, s.f.), esta última fundamental para alejarse del universalismo, profundamente criticado en la actualidad, en tanto metarrelato construido y estructurante de la realidad.

Cabe preguntarse: si lo que plantea el feminismo postcolonial es la articulación de constructos teóricos ya existentes, entonces ¿qué tiene de nuevo? En este caso nos da la impresión que lo novedoso no es en sí su propuesta teórica, sino quien la expresa, cómo la expresa, interpreta, (de)construye, (re)construye $y$ recrea lo ya existente $y$ hace evidente las contradicciones de las teorías feministas, de sus principales exponentes $y$ del territorio $y$ espacio de donde surge y se expresa. Así, la novedad reside en la apropiación teórica y práctica que se ha venido dando por parte de mujeres tercermundistas que, desde ese ser, se apropian y expresan su particularidad y contribuyen con ello al debate $y$ a la construcción del conocimiento.

\section{LA PERTINENCIA DEL ANÁLISIS DE LA VIDA COTIDIANA}

Se observa que a pesar de las distancias conceptuales, Scott (2009), Butler (en Torricella, 2009), Bourdieu (2000) y Chakravorty (1988), señalan al "cuerpo" como uno de los elementos para comprender el género, la desigualdad y las relaciones de poder. Por ello, tomando en cuenta los aspectos conceptuales antes desarrollados, tenemos que preguntarnos dónde observar, o desde dónde observar y explicar los cuerpos y los discursos. Nuestra respuesta es que la reconstrucción de la historia desde lo cotidiano sigue siendo un enfoque metodológicamente adecuado para comprender los discursos, los cuerpos, las relaciones, las prácticas y las representaciones que toman vida en contextos particulares.
La vida cotidiana es una categoría de análisis que se basa en el acontecer de la vida privada y doméstica de las personas, asimismo, aunque las mujeres al incorporarse a espacios públicos salgan en alguna medida de su invisibilidad, su proceso no puede examinarse únicamente desde el ámbito público, ya que es indispensable rescatar y objetivar su vivencia personal como un todo, para entender la complejidad de su proceso. Se conoce que la vida cotidiana de los sujetos se ha venido rescatando como un aspecto central para comprender los procesos que viven $y$ protagonizan las mujeres.

La vida cotidiana surge también del viraje que ha dado la historiografía para encontrar la "otra historia", la historia encubierta de los sujetos, del individuo, que no se hace en los grandes foros o en procesos organizativos formales del ámbito público. Henri Lefebvre es uno de los precursores; incursiona en este campo desde 1946 y en su libro La vida cotidiana en el mundo moderno, de 1980, hace hincapié en la necesidad de vincular la cotidianidad con la sociedad.

Un aspecto que interesa incorporar al análisis de lo cotidiano es la visión de mundo que tienen o han tenido las mujeres de sí mismas y de la sociedad. Para adentrarnos al mundo cotidiano de las mujeres, se debe partir de que "la vida cotidiana como tal es sumamente heterogénea, y esta es la razón por la cual su centro solo puede ser el particular, en el cual aquellas esferas, formas de actividad, etcétera... decididamente heterogéneas se articulan en una Unidad" (Heller, 1987: 93). De esto se puede desprender que la vida cotidiana, a pesar de la particularidad que estudia, no es un valor autónomo, porque son las formas de actividad que se acumulan y se articulan, puesto que a su vez la cotidianidad tiene sentido solo en el contexto de otro medio, en la historia y en los procesos históricos ${ }^{8}$.

8 De acuerdo a la definición que Heller (1972), por vida cotidiana se entiende "... la totalidad de las actividades que caracterizan las reproducciones singulares productoras de la posibilidad permanente de reproducción social". Heller retoma una tesis básica planteada por Marx y Engels en La Ideología Alemana, en la tesis sobre Feuerbach, a saber que "... la transformación de 
La heterogeneidad de los aspectos que componen a la vida cotidiana está determinada por las capacidades físicas del individuo, por sentimientos $y$ emociones que nos involucran como un todo; capacidades y sentimientos que se hacen presentes en la vida cotidiana en la que a su vez, como en la vida pública, tienen lugar procesos de alienación que, aunque distintos, interactúan entre sí.

Se entiende por "cotidianidad" lo que hacemos, sentimos y percibimos de la sociedad, del ámbito familiar, en fin, de las estructuras socioeconómicas de una sociedad determinada, así como, la forma en que actuamos diariamente en ella, en cómo nos vinculamos a los espacios, en lo que somos y queremos ser y la articulación social que se da entre la experiencia individual y la colectiva.

La vida cotidiana de las mujeres (aunque no solo de ellas), incluye muchas formas de sobrevivir y resistir, de subordinarse y rebelarse, que bajo la apariencia de la pasividad o la apatía, cobra otra dimensión o significado; la cultura por ejemplo es un campo de continuidad y ruptura, de aceptación y adaptación, pero también de resistencia y rebelión (Verena, 1986: 94). [Tenemos así que] la vida cotidiana es la totalidad de las actividades que caracterizan las reproducciones singulares de la posibilidad permanente de la reproducción social (Verena, 1986: 9).

La perspectiva cultural estudia la vida cotidiana de las personas en tanto modo de vivir, tratando de vincular y establecer un equilibrio entre el análisis macro y micro social. La visión culturalista posibilita estudiar a las mujeres como personas que se ubican dentro de un conjunto de costumbres, normas, experiencias, percepciones, rituales $y$ acciones, en

la vida cotidiana, de las relaciones y circunstancias de los hombres, no es anterior ni posterior a la transformación política y económica, sino simultánea con ella" (pág. 12). Con ello la autora desea plantear cómo la vida cotidiana es parte de un todo integrado, por tanto, las acciones que se realizan cotidianamente responden a un contexto histórico específico. donde se expresan y experimentan actividades y relaciones sociales.

Toda persona tiene que vivir la vida cotidiana. Nadie, independientemente de su posición social, del lugar que ocupa en la división social del trabajo, puede desprenderse totalmente de su cotidianidad. Por eso, Heller afirma que la vida cotidiana es la vida de toda persona (Heller: 1972). Más ello no significa que la vida de una persona ${ }^{9}$ esté totalmente determinada por la vida cotidiana; en tanto, en la vida cotidiana las personas participan de forma integral, movilizando “...[t]odas sus capacidades intelectuales, sus habilidades manipulativas, sus sentimientos, pasiones, ideas e ideologías" (Heller, 1972: 39). Agrega al respecto que "el hombre de la cotidianidad es activo y goza, obra $y$ recibe, es afectivo y racional, pero no tiene ni tiempo ni posibilidad de absorberse enteramente en ninguno de estos aspectos para poder apuntarlo según toda su intensidad" (Heller, 1972: 40).

La cotidianidad precede al sujeto. Las personas nacen insertas en su cotidianidad. Mediante la socialización, el sujeto aprende las habilidades necesarias para la vida cotidiana en la sociedad. Más ello no significa que no pueda modificar algunas de sus prácticas socialmente aprendidas. La persona adulta debe ser capaz de "manipular las cosas" imprescindibles para la vida cotidiana. El proceso de asimilación de las cosas y las relaciones sociales - lo que otras escuelas denominarían socialización- empieza siempre "por grupos" (ejemplo, la familia, escuela o comunidades menores). Estos grupos - copresenciales- median y transmiten al individuo las costumbres, las normas y la ética. Existe así una relación entre los "predecesores", los "contemporáneos" y los "sucesores", una persona en particular integra parte de las experiencias que le han sido transmitidas en el tiempo en sus acciones $y$ forma de vida, ya sea

9 La autora en el texto no habla de la persona sino del hombre; sin embargo, hemos considerado que la palabra persona o individuo(a) es más inclusiva que la de hombre, en la medida en que esta última nos puede a veces inducir a mirar solo a varones, dejando por fuera a las mujeres. 
por sus contemporáneos, los predecesores o bien, sucesores ${ }^{10}$.

Según Heller (1972), Bourdieu (2000) y Goffman (1997), lo más relevante es que los elementos que constituyen y caracterizan la vida cotidiana no producen "absolutos". Siempre existe un margen de acción, movimiento y posibilidad de desarrollo para el individuo. Existen las contradicciones, razón por la cual es posible la presencia de resistencias, movimientos contraculturales y contranormativos (León, 1999). Si las personas no cuentan con dicho espacio de acción se produce la "extrañación". Según Heller, la extrañación ocurre cuando en lo cotidiano la persona no tiene espacio, campo, libertad alguna de elección o selección, posibilidades de desarrollo. En tanto que “... extrañación, es, desde luego, siempre extrañación respecto de algo, $y$ precisamente respecto de las posibilidades concretas del desarrollo específico de la humanidad" (Heller, 1972: 42).

\section{CONCLUSIONES}

Los aspectos desarrollados en este artículo pueden contribuir a delimitar nuestro ángulo analítico. Teórica y metodológicamente se destaca la importancia que siguen teniendo el análisis del discurso y del cuerpo para los estudios de género y para las mujeres, así como para las temáticas sociales generales, en la medida en que permiten analizar las relaciones de poder que nos sitúan en el terreno de las desigualdades socio históricas y los cambios que se producen de la confrontación, el conflicto, las resistencias, entre otras acciones que devienen de los sujetos, así como también comprender las estructuras y esquemas sociales que las mantienen y las hacen trasponibles en el tiempo y en espacios diversos. El discurso, tal y como lo vienen señalando algunas teóricas del feminismo, refleja sistemas de significados al igual que el lenguaje, asimismo, permite observar en el tiempo las representaciones que se generan a partir de él. Es central retomar la forma en que Scott establece la relación entre lenguaje y discurso:

10 Consultar: Schutz y Luckmann. The Structures of the Life-World, Vol.2, 1989.
El lenguaje opera no como simple medio de comunicación, sino como discurso. Entendiendo por discurso, no frases o palabras, "sino formas totales de pensamiento, de comprensión de cómo opera el mundo, $y$ de cuál es el lugar que uno tiene en él" (Cabrera, 2006: 22).

Es preciso considerar también el cuerpo como constructo simbólico, tomando en cuenta los aportes de Foucault ${ }^{11}$, Bourdieu ${ }^{12}$ y Csordas $^{13}$. Considerar a la persona como agente encarnado busca romper con las principales dualidades del pensamiento occidental: mente/ cuerpo, sujeto/objeto, objetivo/sujetivo, pasivo/ activo, racional/emocional, lenguaje/experiencia, lo que nos aproxima de nuevo a la crítica que se ha venido señalando al concepto del género, el uso y abuso del mismo, que ha llevado a enfatizar la visión dicotómica del mundo, que a su vez lo fragmenta $y$ acentúa las diferencias físicas, los roles sociales, etc. La idea de la diferencia sexual, de los géneros, etc., es la que al fin y al cabo está en función de nuestro conocimiento sobre el cuerpo y "ese conocimiento no es puro, no puede ser aislado de su implicación en una amplia gama de contextos discursivos" (Cabrera, 2006).

$11 \quad$ Al respecto no se pueden dejar de citar los aportes de autores que son claves en este aspecto conceptual: Foucault, quien desde el concepto de biopoder, relaciona la experiencia del cuerpo con el poder, el conocimiento y los cambios políticos que se han dado en la historia con respecto al cuerpo. El cuerpo es visto como la experiencia material sobre la que se ejerce poder, pero también permite resistir a los sujetos sociales. En: Foucault, Michel. La historia de la sexualidad I, La voluntad de saber. España: Siglo XXI, 1987.

12 Bourdieu (2000 y 1990) lo utiliza para comprender los procesos socio culturales que entrañan las prácticas y habitus de las personas, asimismo, lo utiliza en el estudio de las clases sociales, los gustos, la dominación masculina, entre otras.

13 Csordas (s.f.) quien habla de embodiment y bodilyness (corporalidad) para referirse a lo corporal como auténtico campo de la cultura. El cuerpo es considerado un agente y lugar de intersección individual, psicológica y social, es ser biológico pero también es entidad consciente, experiencial, actuante e interpretadora. 
Por su parte, el estudio de la cotidianidad permite comprender el "discurso" y el "cuerpo" como un medio de vinculación de lo personal con lo sociohistórico, para desde allí generar el contexto que permita entender al sujeto, su lenguaje y su cuerpo, que son manifestación y expresión de las relaciones de género. Así, la relación del binomio individuosociedad pierde su contenido dicotómico y pasa a formar parte de un todo: ambos se retroalimentan mutuamente para producir $y$ reproducir a la persona, a la sociedad. Cuando se considera el género como categoría de análisis social y/o histórica, el reto consiste en ir más allá de lo previsible sobre las diferencias en el estatus $y$ las experiencias de hombres $y$ mujeres, pues se cae en la mera diferenciación de los roles y las vivencias... Retomando a Scott, se debe estudiar cómo los significados que expresan hombres y mujeres a su vez, expresan discursos de poder o establecidos $y$ naturalizados (Cabrera, 2006); de ahí las contradicciones que las personas muestran con respecto a sus discursos, o bien, entre los discursos y las prácticas. No todo es producción reflexiva, pero tampoco es reproducción acríti$\mathrm{ca}$, ahistórica o inercial.

Para estudiar la historia de las mujeres $0 / y$ la historia del género, es indispensable estudiar los "pliegues de la historia" de los que habla Foucault; encontrar —en el camino no lineal de las acciones humanas- lo que no se ha dicho, lo que no se ha escuchado, lo que al fin $y$ al cabo, la historia oficial suele dejar de lado pero prevalece en el entretejido social, porque ha existido y existe por medio de quienes asistieron, observaron, protagonizaron y contaron la otra historia, la aún no contada.

\section{REFERENCIAS BIBLIOGRÁFICAS}

Alcoff, Linda. "Feminismo cultural versus pos-estructuralismo: la crisis de la identidad en la teoría feminista". Revista Feminaria 4. Universidad de Buenos Aires, noviembre 1989.

Amorós, Celia. Tiempo de feminismo. Sobre feminismo, proyecto ilustrado $y$ posmodernidad. 2da. edición. Madrid: Ediciones Cátedra, 2000.
Aresti, Nerea. "La categoría de género en la obra de Joan Scott". Joan Scott y las politicas de la historia. En: Joan Scott. España: Icaria Editorial, 2006.

Arreaza, Catalina y Tickner, Arlene B. "Postmodernismo, postcolonialismo y feminismo: manual para (in) expertos". Revista Colombia Internacional 2 (54). 2002: 14-38.

Benhabib, Seyla. "Desde las políticas de identidad al feminismos social: un alegato para los noventa". Las ciudadanas y lo político. España: Instituto Universitario de Estudios de la Mujer, 1996.

Blanco, Graciela. "Las desigualdades entre los sexos en la Universidad de Costa Rica". La mujer en la Universidad, el caso centroamericano. Ligia Delgadillo (comp.). Costa Rica: Editorial GuayacanILIFEM, s.f.

Bock, Gisela. "El lugar de las mujeres en la historia”. Revista Sociológica 10. Mayoagosto 1989.

Borderías, Cristina (ed.). "Prólogo". Joan Scott y las políticas de la historia. Madrid: Icaria Editorial, 2006.

Bourdieu, Pierre. "Structures, habitus, practices". The logic of practice. United Kingdom: Polity Press (United States), 1990.

Bourdieu, Pierre. La dominación masculina. 2da edición. México: Editorial ANAGRAmA, 2000.

Butler, Judith. El género en disputa, el feminismo y la subversión de la identidad. México: Paidós-Universidad Nacional Autónoma de México-Programa Universitario de Estudios de Género, 2001.

Butler, Judith (1996). "El marxismo y lo meramente cultural". New Left Review 2. Mayo-junio 2008: 109-22.

Cabera, Miguel Ángel. "Lenguaje, experiencia e identidad. La contribución de Joan Scott a la renovación teórica de los estudios de históricos". Joan Scott y las politicas de la historia. España: Icaria Editorial, 2006. 
Carrera, Isabel. "Feminismo y postcolonialismo: estrategias de subversión”. Universidad de Oviedo. s.f. En: <http://www.bivipas. info/bitstream/10720/466/1/D-352Carrera_Isabel-276.pdf.>

Comisión Económica para América Latina y el Caribe (CEPAL). Mujeres en América Latina. México: Fondo de Cultura Económica, s.f.

Chakrabarty, Dipesh. "Subaltern studies and postcolonial historiography". Nepantla. Views from South 1 (1). Durham. Duke University Press, 2000: 9-32.

Contreras, Gerardo. "Medio siglo de la educación superior en Costa Rica". Káñina. Revista de Artes y Letras XV (1-2). Costa Rica: EUCR, 1991.

Csordas, Thomas. "Introduction: the body as representation and being-in-theword". Embodiment and experience the existential ground of culture and self. Thomas Csordas (ed.). Cambridge. Cambridge University Press, s.f.: 1-24.

De la Pascua, María José. "Comentario al libro por Mary Nash”. Mujeres solas: historia de amor y abandono en el mundo hispánico. lera edición. España: Editorial Servicio de publicaciones-Centro de Ediciones de la Diputación de Málaga, 1998.

De Lauretis, Teresa. Technologies of gender: essays on theory film and fiction. lera edición. Estados Unidos: Indiana University Press, 1987.

Eisenstein, Zillah. "Hacia el desarrollo de una teoría del patriarcado capitalista y el feminismo socialista”. Teoría feminista. lera edición. República Dominicana: Ediciones Populares Feministas, 1984.

Goffman, Erving (1959). La presentación de la persona en la vida cotidiana. Buenos Aires: Amorrortu editores, 1997

Hawkesworth, Mary. "Confundir el género". Debate Feminista 20. Año 10. México, octubre 1999.

Heller, Ágnes. La revolución de la vida cotidiana. España: Editorial Península, 1987.

Heller, Ágnes. Historia y vida cotidiana: aportación de la sociología socialista. 1era edición. España: Editorial Grijalbo, 1972.

Izquierdo, María Jesús. El malestar en la desigualdad. lera. edición. España: Ediciones Cátedra-Universidad de Valencia-Instituto de la Mujer, 1998.

Lagarde, Marcela. Género y Feminismo. Desarrollo Humano y democracia. $2 \mathrm{da}$. edición. España: Ed. Horas y Horas, 1997.

Lagarde, Marcela. Los cautiverios de las mujeres: madresposas, monjas, putas, presas y locas. Colección Posgrado. México: Universidad Nacional Autónoma de México, 1993.

León, Emma. Usos y discursos teóricos sobre la vida cotidiana. México: Universidad Autónoma de MéxicoAnthropos Editorial-Centro Regional de Investigaciones Multidisciplinarias, 1999.

Nash, Mary. Presencia y protagonismo: aspectos de la historia de la mujer. Barcelona: Serbal, 1984.

Piedra, Nancy. "Feminismo y postmodernidad: entre el ser para sí o el ser para los otros". Revista Ciencias Sociales 101-102 (III-IV). Costa Rica: EUCR, 2003.

Radkau, Verena. "Hacia una historiografía de la mujer”. Revista Nueva Antropología 30 (VIII). México 1986: 77-94.

Ramos, Carmen. Género e historia: la historiografía sobre la mujer. México: Instituto Mora -Universidad Autónoma Metropolitana, 1992.

Riquer, Florinda. "La identidad femenina en la frontera entre la conciencia y la interacción social". La voluntad de ser: las mujeres en los noventa. lera edición. México: El Colegio de México, 1997.

Salles, Vannia. "Una discusión de aportes y conceptos con base en la revisión de textos clave sobre género". Mimeo. México: El Colegio de México-Centro de Estudios Sociológicos, 1999.

Salles, Vannia. "Sociología de la cultura, relaciones de género y feminismos: una revisión de aportes". Estudios sobre las mujeres y las relaciones de género 
en México: aportes desde diversas disciplinas. México: El Colegio de México, 2002.

Scott, Joan. "El movimiento por la paridad: un reto al universalismo francés". Joan Scott y las políticas de la historia. Cristina Borderías (ed.). Madrid: Icaria Editorial, 2006.

Sharrat, Sara. "Feminismo y ciencia: una relación problemática". Cuadernos de Ciencias Sociales 65. Costa Rica: Facultad Latinoamericana de Ciencias Sociales (FLACSO), 1993.
Tarres, María Luisa. "Prólogo". La voluntad de ser: las mujeres en los noventa. lera edición. México: El Colegio de México, 1997.

Vigil, Mariló. La vida de las mujeres en los siglos XVI y XVII. Madrid: Siglo XXI, s.f.

Zemon, Natalie. Women's history in transition: the European case. Inglaterra: Feminist Studies Inc., 1976.

Fecha de ingreso: $22 / 07 / 2010$

Fecha de aprobación: 20/03/2013 